\title{
MÉTODOS NÃO FARMACOLÓGICOS PARA O TRATAMENTO DO ALZHEIMER: UMA REVISÃO INTEGRATIVA
}

\author{
NON-PHARMACOLOGICAL METHODS FOR ALZHEIMER 'S TREATMENT: AN INTEGRATING REVIEW
}

\section{METOdOS NO FARMACOLÓGICOS PARA EL TRATAMIENTO DEL ALZHEIMER: UNA REVISIÓN INTEGRADORA}

Bruna Guedes Lopes Costa ${ }^{1}$, Luciano Ramos de Lima ${ }^{2}$, Silvana Schwerz Funghetto ${ }^{3}$, Cris Renata Grou Volpe ${ }^{4}$, Walterlania Silva Santos ${ }^{5}$, Marina Morato Stival ${ }^{6}$.

\section{RESUMO}

Objetivo: identificar, na literatura, as propostas de métodos não farmacológicos para o tratamento da doença de Alzheimer. Métodos: Trata-se de uma revisão integrativa realizada em três bases de dados. Foram incluídos artigos publicados entre os anos de 2005 a 2016, na íntegra, nos idiomas português, inglês e espanhol. Foram adotados os descritores: idoso, terapêutica e doença de Alzheimer. Os artigos foram analisados pelo título e resumo, estratificados pela base de dados, periódico, ano, país, título, tipo de estudo, sujeitos, local pesquisa e nível de evidência. Posteriormente, foi realizada uma síntese do conhecimento obtido sobre os métodos não farmacológicos utilizados no tratamento da doença de Alzheimer. Resultados: Dez estudos foram analisados na pesquisa, sendo que as principais intervenções não farmacológicas identificadas foram $50 \%$ na atividade motora, $20 \%$ atividade cognitiva, $20 \%$ musicoterapia e $10 \%$ terapia multicomponente. Conclusão: Os tratamentos não farmacológicos provaram ser eficientes, pois reduziram a dependência para a realização de atividades de vida diária e, consequentemente, melhoraram a qualidade de vida de pacientes com doença de Alzheimer.

Descritores: Idoso; Doença de Alzheimer; Terapêutica.

\begin{abstract}
Objective: to identify in the literature proposals for non-pharmacological methods for the treatment of Alzheimer's disease. Methods: This is an integrative review performed in three databases. We included articles published between 2005 and 2016 , in full, in Portuguese, English and Spanish. It was adopted the descriptors of the elderly people, therapeutic and Alzheimer's disease. The articles were analyzed by the titles and abstracts, and were stratified by database, journal, year, country, title, type of study, subjects and local research and level of evidence. Subsequently, a synthesis of the knowledge obtained on the non-pharmacological methods used in the treatment of Alzheimer's disease was performed. Results: Ten studies were analyzed and nonpharmacological interventions identified were in $20 \%$ in motor activity, $20 \%$ in cognitive activity, $20 \%$ in music therapy and $10 \%$ in multicomponent therapy. Conclusion: The non-pharmacological treatments have proven to be effective. They reduced the dependence to perform activities of daily living and, consequently, improved the quality of life of patients with Alzheimer's disease. Descriptors: Aged; Alzheimer disease; Therapeutics.
\end{abstract}

\section{RESUMEN}

Objetivo: Identificar, en la literatura, propuestas de métodos no farmacológicos para el tratamiento de la enfermedad de Alzheimer. Métodos: Esta es una revisión integradora realizada en tres bases de datos. Incluimos artículos publicados entre 2005 y 2016, en su totalidad, en portugués, inglés y español. Se adoptaron las siguientes palabras clave: ancianos, terapéuticos y enfermedad de Alzheimer. Los artículos fueron analizados por título y resumen, estratificados por base de datos, revista, año, país, título, tipo de estudio, temas, lugar de investigación y nivel de evidencia. Posteriormente, se realizó una síntesis de los conocimientos obtenidos sobre los métodos no farmacológicos utilizados en el tratamiento de la enfermedad de Alzheimer. Resultados: Se analizaron diez estudios en la investigación y las principales intervenciones no farmacológicas identificadas fueron: $50 \%$ en actividad motora, $20 \%$ de actividad cognitiva, $20 \%$ de musicoterapia y $10 \%$ de terapia multicomponente. Conclusión: Los tratamientos no farmacológicos demostraron ser eficientes, ya que redujeron la dependencia de las actividades de la vida diaria y, en consecuencia, mejoraron la calidad de vida de los pacientes con enfermedad de Alzheimer.

Descriptores: Anciano; Enfermedad de Alzheimer; Terapéutica.

${ }^{1}$ Enfermeira. Residente em Enfermagem na Secretaria de Estado de Saúde do Distrito Federal. ${ }^{2}$ Enfermeiro. Doutorando em Ciências e Tecnologias da Saúde pela Faculdade de Ceilândia/Universidade de Brasília. ${ }^{3}$ Enfermeira. Doutora em Ciências da Saúde pela Universidade de Brasília. Docente do Curso Pós-Graduação em Ciências e Tecnologias da Saúde da Faculdade de Ceilândia/Universidade de Brasília. ${ }^{4}$ Enfermeira. Doutora em Enfermagem pela Universidade de Brasília. Docente do Curso de Enfermagem da Faculdade de Ceilândia/Universidade de Brasília. ${ }^{5}$ Enfermeira. Doutora em Ciências da Saúde Universidade Federal de Goiás . Docente do Curso de PósGraduação em Enfermagem da Universidade de Brasília. ${ }^{6}$ Enfermeira. Doutora em Ciências e Tecnologias da Saúde pela Faculdade de Ceilândia/Universidade de Brasília. Docente do Curso de Enfermagem da Faculdade de Ceilândia/Universidade de Brasília. 


\section{INTRODUÇÃO}

A Doença de Alzheimer (DA) consiste na diminuição da função cerebral, caracterizada pela perda progressiva das funções cognitivas e da memória, afetando o indivíduo em suas atividades de vida diária (AVD) e atividades instrumentais de vida diária (AIVD), ocasionando perda da autonomia ${ }^{(1)}$.

A ocorrência de demências, como a DA, afeta $55 \%$ da população acima de 65 anos, sendo que as demências foram consideradas a sexta causa de morte nos países desenvolvidos em 2004 e a quinta causa de morte em 2006, ou seja, países com maior população idosa. Essa transição demográfica também ocorre no Brasil, principalmente, em razão da diminuição da mortalidade, da fecundidade e à melhora na expectativa de vida. Nota-se um aumento substancial da população idosa, o que amplia a probabilidade de ocorrência de demências como a DA que afeta $55 \%$ da população acima de 65 $\operatorname{anos}^{(2)}$.

Atualmente, no mundo, 35,6 milhões de pessoas vivem com a DA. A estimativa é que esse número praticamente dobre a cada 20 anos, chegando a 65,7 milhões em 2030 e 115,4 milhões em 2050. No Brasil, não há dados sobre a incidência da DA, entretanto, segundo pesquisas, estimam-se que cerca de 1,2 milhão de pessoas sofram com a doença ${ }^{(2-4)}$.

A DA pode ser dividida em três fases - leve, moderada ou grave - caracterizadas pelo seu nível de comprometimento cognitivo e o seu grau de dependência. $\mathrm{Na}$ primeira fase, são identificados um comprometimento da memória recente e desorientação de tempo e espaço e dura em média 2 a 3 anos. Na fase intermediária que duram de 2 a 10 anos, são percebidos comprometimento da memória remota, dificuldades em resolver problemas e atividades operativas, afetando as atividades básicas e instrumentais da vida diária. E na fase final, com duração de 8 a 12 anos, ocorre total dependência, evidenciada pela perda da capacidade para realizar atividades básicas e instrumentais e imobilidade ${ }^{(1)}$.

O diagnóstico da DA só pode ser estabelecido a partir de um quadro clínico e exclusão de outros casos de demência por meio de exames laboratoriais e neuropatológicos. Quanto ao tratamento, a DA ainda não possui cura, entretanto existem medidas farmacológicas que visam a reduzir os efeitos cognitivos e de memória, sendo estes baseados na prescrição de anticolinesterásicos (rivastigmina, donepezil e galantamina) e de memantina (antiglutamatérgico) $^{(5)}$.

Além disso, podem ser utilizadas medidas não farmacológicas que visam a proporcionar uma melhora na qualidade de vida do paciente e de sua família/cuidador. As quais têm como proposta estabelecer o uso mais eficiente da memória, por meio de estratégias de atendimento multidisciplinar, com técnicas mnemônicas ou de aprendizagem, estratégias compensatórias, terapias de orientação da realidade e a abordagem terapêutica com grupos de famílias e cuidadores ${ }^{(6)}$.

O Departamento Científico de Neurologia Cognitiva e do Envelhecimento da Academia Brasileira de Neurologia realizou uma revisão de literatura a fim de sintetizar recomendações e sugestões para o Tratamento da DA, evidenciando a importância do tratamento tanto farmacológico, como do tratamento não farmacológico - do comprometimento cognitivo por meio de técnicas de reabilitação cognitiva, treinamento cognitivo de habilidades específicas (memória e linguagem) e técnicas visando a melhora nas AVDs; e dos sintomas de comportamento e psicológicos, por meio de programas educacionais e treinamento de cuidadores, música e passeios ${ }^{(7)}$.

No âmbito internacional, um estudo foi realizado com o objetivo de avaliar a qualidade de vida de pacientes com diagnóstico de DA que foram submetidos à terapia assistida com animais. Os resultados demonstraram que, ao final da terapia, $100 \%$ dos participantes apresentaram melhora nos aspectos físicos, comportamentais e psicológicos ${ }^{(8)}$.

Os estudos que abordam a DA como tema de pesquisa vêm aumentando, porém com um enfoque no tratamento farmacológico. Diante da escassez de investigações e das incertezas sobre a utilização dos métodos não farmacológicos no tratamento para DA, a importância em realizar este estudo deve-se ao fato de que a DA é uma degeneração que acomete idosos e que não tem cura, e com o tratamento não farmacológico pode-se viabilizar maneiras de melhorar a qualidade de vida desse paciente, de forma que possa integrar a participação do familiar/cuidador no tratamento. Além disso, conhecer os métodos não farmacológicos para o tratamento dessa doença poderá nortear os profissionais de saúde envolvidos nesse contexto. 
Diante do exposto o objetivo deste estudo foi identificar, na literatura, as propostas de métodos não farmacológicos para o tratamento da doença de Alzheimer (DA).

\section{MÉTODO}

Trata-se de uma revisão integrativa (RI) da literatura que permite um apanhado de estudos científicos realizados sobre determinado tema, a fim de comprovar efetividade deste e correlacionar a sua aplicabilidade na prática clínica $^{(9)}$.

A RI consiste em método de pesquisa dividido em seis etapas. Na primeira etapa, ocorreu a identificação do tema e elaboração da questão norteadora, por meio da estratégia PICO (Paciente/problema, Intervenção, Comparação e Outcomes-desfecho) $^{(9)}$, descritos com os seguintes componentes: $\mathrm{P}$ - pacientes idosos com doença de Alzheimer; I métodos/terapêutica não farmacológicos utilizados no tratamento da doença de Alzheimer; C - relação de benefício existente entre os outros tratamentos; $\mathrm{O}$ - controle da doença ou melhora da qualidade de vida do idoso. Assim, emergiu a seguinte questão norteadora: "Quais são os métodos/terapêutica não farmacológicos utilizados no tratamento do idoso com doença de Alzheimer?".

$\mathrm{Na}$ segunda etapa, do estudo foram estabelecidos os critérios de inclusão e exclusão dos estudos. Foram adotados os seguintes critérios de inclusão: a abrangência temporal entre os anos de 2005 a 2016, os artigos deveriam estar disponíveis na íntegra para leitura nos idiomas português, inglês ou espanhol e deveriam abordar o tema de estudo. Os critérios de exclusão foram: artigos editoriais, comentários ou críticas, artigos de revisão de literatura e artigos que não apresentavam relação com o tema investigado.

A busca de artigos foi realizada nos meses de outubro de 2017 a dezembro de 2017, nas bases de dados Lilacs, Pubmed e Scielo, considerando os seguintes Descritores em Ciências da Saúde (DeCS): "idoso", "aged", "terapêutica", "Therapeutics", "doença de Alzheimer", "Alzheimer Disease".

Os artigos inicialmente foram analisados pelo título e resumo. Após essa primeira análise, procedeu-se com a terceira etapa do estudo, na qual foram definidas as informações a serem extraídas dos estudos selecionados. A fim de melhor organização, foram extraídas dos estudos as seguintes informações: base de dados, periódico, ano, país, título, tipo de estudo, nível de evidência (foi estabelecido pelo critério Oxford Centre Evidence-Based Medicine) ${ }^{(10)}$ e tipo de método não farmacológico utilizado na pesquisa. Posteriormente, foram organizados e apresentados de acordo com a autoria, objetivo do estudo, desenho metodológico, idioma.

Os estudos analisados foram classificados de acordo com o nível de evidência: Nível 1 revisões sistemáticas ou metanálise de relevantes ensaios clínicos; Nível 2 - evidências derivadas de pelo menos um ensaio clínico randomizado controlado bem delineado; Nível 3 - ensaios clínicos bem delineados sem randomização; Nível 4 - estudos de coorte e de caso-controle bem delineados; Nível 5 - revisão sistemática de estudos descritivos e qualitativos; Nível 6 evidências derivadas de um único estudo descritivo ou qualitativo; Nível 7 - opinião de autoridades ou relatório de comitês de especialistas $^{(10)}$.

$\mathrm{Na}$ quarta etapa, foram analisados os estudos selecionados e incluídos na RI. Nesse momento da pesquisa, foi realizada uma análise crítica e detalhada de todos os artigos selecionados. Na quinta etapa, houve a interpretação dos resultados. Na última etapa, ocorreu a apresentação da revisão com a síntese do conhecimento obtido sobre os métodos não farmacológicos utilizados no tratamento da doença de Alzheimer. A análise dos estudos selecionados foi realizada tanto no que se refere à síntese dos dados extraídos dos artigos quanto na possibilidade de descrever e classificar os dados, com o intuito de reunir o conhecimento produzido sobre o tema explorado nesta revisão.

$\mathrm{Na}$ Figura 1, apresenta-se o caminho percorrido para seleção de artigos desta RI. 
Figura 1 - Seleção dos estudos incluídos na síntese. Brasília, 2017.
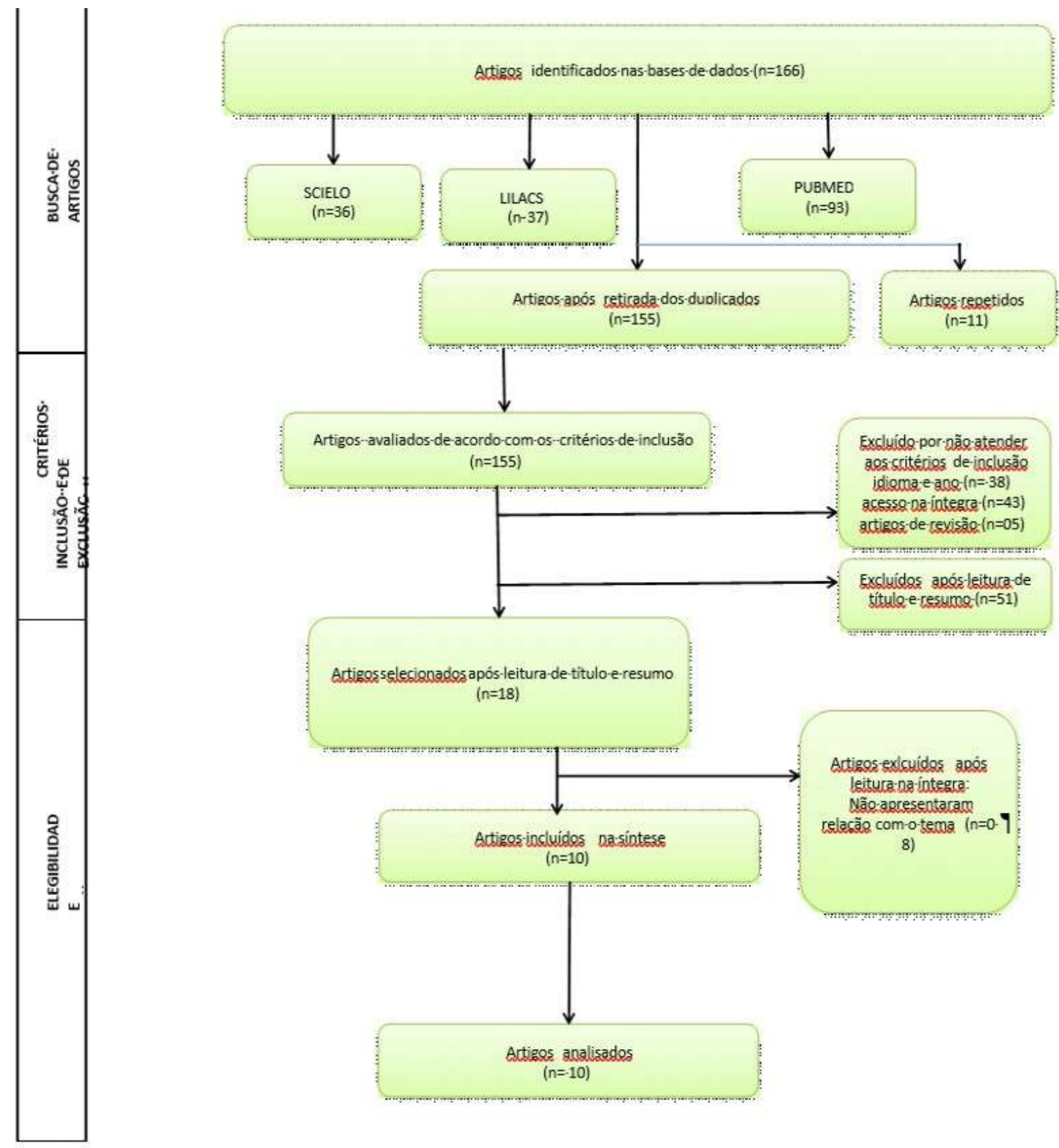

Fonte: Elaborado pelos autores.

\section{RESULTADOS E DISCUSSÃO}

\section{Caracterização dos estudos}

No Quadro 1, os estudos estão distribuídos de acordo com os autores, título do artigo, base de dados, periódico em que foi publicado e o ano de publicação. Em relação ao ano de publicação, verificou-se um predomínio de estudos, no ano de 2011, com quatro estudos, seguido por 2008 com dois estudos. O maior número de publicação deu-se no periódico Clínica com três estudos e

Arquivo de Neuro- Psiquiatria com dois estudos, sendo que, de forma equilibrada, metade dos estudos estavam entre revistas nacionais e internacionais respectivamente. Apenas dois estudos foram publicados em revistas específicas de enfermagem (Quadro 1). 
Quadro 1- Distribuição dos estudos incluídos na revisão integrativa segundo autores, título, base de dados, periódicos e ano de publicação. Brasília, 2017.

\begin{tabular}{|c|c|c|c|c|c|}
\hline № & Autores & Título & $\begin{array}{l}\text { Base de } \\
\text { Dados }\end{array}$ & Periódicos & Ano \\
\hline 01 & $\begin{array}{c}\text { Arcoverde C. et } \\
\text { al. }^{(11)}\end{array}$ & $\begin{array}{l}\text { Treadmill training as an augmentation } \\
\text { treatment for Alzheimer's disease: a pilot } \\
\text { randomized controlled study }\end{array}$ & SciELO & $\begin{array}{l}\text { Arquivo de } \\
\text { Neuro- } \\
\text { Psiquiatria. }\end{array}$ & 2014 \\
\hline 02 & $\begin{array}{c}\text { Arcoverde C. et } \\
\text { al. }{ }^{(12)}\end{array}$ & $\begin{array}{c}\text { Role of physical activity on the } \\
\text { maintenance of cognition and activities of } \\
\text { daily living in elderly with Alzheimer's } \\
\text { disease }\end{array}$ & SciELO & $\begin{array}{l}\text { Arquivo de } \\
\text { Neuro- } \\
\text { Psiquiatria. }\end{array}$ & 2008 \\
\hline 03 & $\begin{array}{c}\text { Hernandez SSS. } \\
\text { et al. }^{(13)}\end{array}$ & $\begin{array}{l}\text { Efeitos de um programa de atividade física } \\
\text { nas funções cognitivas, equilíbrio e risco } \\
\text { de quedas em idosos com demência de } \\
\text { Alzheimer. }\end{array}$ & Lilacs & $\begin{array}{l}\text { Revista } \\
\text { Brasileira de } \\
\text { Fisioterapia }\end{array}$ & 2010 \\
\hline 04 & $\begin{array}{l}\text { Albuquerque } \\
\text { MCS. et al }{ }^{(14)}\end{array}$ & $\begin{array}{c}\text { Os efeitos da música em idosos com } \\
\text { doença de Alzheimer de uma instituição } \\
\text { de longa permanência }\end{array}$ & Lilacs & $\begin{array}{c}\text { Revista } \\
\text { Eletrônica de } \\
\text { Enfermagem }\end{array}$ & 2012 \\
\hline 05 & Viola LF. et al ${ }^{(15)}$ & $\begin{array}{l}\text { Effects of a multidisciplinar cognitive } \\
\text { rehabilitation program for patients with } \\
\text { mild Alzheimer's disease }\end{array}$ & Lilacs & Clinis & 2011 \\
\hline 06 & Stella F. et al. ${ }^{(16)}$ & $\begin{array}{l}\text { Attenuation of neuropsychiatric } \\
\text { symptoms and caregiver burden in } \\
\text { Alzheimer's disease by motor } \\
\text { intervention: a controlled trial }\end{array}$ & Lilacs & Clinics & 2011 \\
\hline 07 & $\begin{array}{l}\text { Christofoletti G. } \\
\text { et al. }^{(17)}\end{array}$ & $\begin{array}{c}\text { Physical activity attenuates } \\
\text { neuropsychiatric disturbances and } \\
\text { caregiver burden in patients with } \\
\text { demential }\end{array}$ & Lilacs & Clinics & 2011 \\
\hline 08 & $\begin{array}{c}\text { Souza PA. et } \\
\text { al. }^{(18)}\end{array}$ & $\begin{array}{l}\text { Oficinas de estimulação cognitiva para } \\
\text { idosos com demência: uma estratégia de } \\
\text { cuidado na enfermagem gerontológica }\end{array}$ & Lilacs & $\begin{array}{l}\text { Revista Gaúcha } \\
\text { de Enfermagem }\end{array}$ & 2008 \\
\hline 09 & $\begin{array}{l}\text { Sakamoto M. et } \\
\text { al. }^{(19)}\end{array}$ & $\begin{array}{l}\text { Comparing the effects of different } \\
\text { individualized music interventions for } \\
\text { elderly individuals with severe dementia }\end{array}$ & Pubmed & $\begin{array}{l}\text { International } \\
\text { Psychogeriatrics } \\
\text { / IPA }\end{array}$ & 2013 \\
\hline 10 & $\begin{array}{c}\text { Graessel E. et } \\
\text { al. }^{(20)}\end{array}$ & $\begin{array}{l}\text { Non-pharmacological, multicomponent } \\
\text { group therapy in patients with } \\
\text { degenerative dementia: a } 12 \text {-month } \\
\text { randomizied, controlled trial }\end{array}$ & Pubmed & BMC Medicine & 2011 \\
\hline
\end{tabular}

Fonte: Elaborado pelos autores para fins deste estudo.

De acordo com o Quadro 2, a distribuição por tipo de estudo, quatro são controlados randomizados, sendo três simples cego e um duplo-cego, e três são controlados sem randomização. Em relação ao país de origem da publicação, identificou-se uma maior incidência de estudos no Brasil, com sete publicações. Desses estudos analisados, sete foram publicados no idioma inglês e apenas três foram publicados no idioma português. De acordo com o nível de evidência, quatro estudos obtiveram o nível 2, três estudos apresentaram o nível 3 , dois estudos com nível 4 e apenas um estudo com nível de evidência 6 .

A maioria dos estudos foi realizada com idosos com diagnóstico de DA do tipo leve a moderada, sendo 3 em Institutos de Psiquiatria de Universidades Federais. Quanto ao método não farmacológico utilizado nos estudos houve um predomínio da atividade física ou exercício aeróbio com cinco estudos, a intervenção cognitiva ou reabilitação cognitiva esteve presente em dois estudos, assim como a musicoterapia que também esteve presente em 
dois estudos, seguidos da terapia MAKS (Estimulação motora, atividades de vida diária, estimulação cognitiva e elemento espiritual) em apenas um estudo (Quadro 2).

Quadro 2 - Distribuição dos estudos incluídos na revisão integrativa segundo o tipo de estudo, nível de evidência, sujeitos, local, país, idioma e método não farmacológico utilizado na pesquisa. Brasília, 2017.

\begin{tabular}{|c|c|c|c|c|c|}
\hline № & Tipo de Estudo & $\begin{array}{l}\text { Nível de } \\
\text { Evidência }\end{array}$ & Sujeitos/Local & País & $\begin{array}{l}\text { Método não } \\
\text { farmacológico }\end{array}$ \\
\hline 01 & $\begin{array}{l}\text { Estudo piloto } \\
\text { controlado, } \\
\text { randomizado e } \\
\text { simples-cego }\end{array}$ & 2 & $\begin{array}{l}\text { Idosos }(n=20) \text { sendo } 16 \text { com DA e } 4 \text { com } \\
\text { Demência Mista/Instituto de Psiquiatria da } \\
\text { Universidade Federal do Rio de Janeiro. }\end{array}$ & Brasil & Atividade Física \\
\hline 02 & $\begin{array}{l}\text { Estudo Analítico, } \\
\text { Observacional e } \\
\text { Transversal }^{12}\end{array}$ & 4 & $\begin{array}{l}\text { Idosos ( } n=37 \text { ) com DA leve a moderada com } \\
\text { mais de } 65 \text { anos de idade/ Instituto de } \\
\text { Psiquiatria da Universidade Federal do Rio de } \\
\text { Janeiro e Open University - Terceira Idade }\end{array}$ & Brasil & Atividade Física \\
\hline 03 & $\begin{array}{l}\text { Estudo controlado, } \\
\text { sem randomização }\end{array}$ & 3 & $\begin{array}{c}16 \text { Idosos com diagnóstico de DA leve a } \\
\text { moderada./ Comunidade de Rio Claro (SP), } \\
\text { Brasil. }\end{array}$ & Portugal & Atividade Física \\
\hline 04 & $\begin{array}{l}\text { Estudo descritivo- } \\
\text { exploratório de } \\
\text { abordagem } \\
\text { qualitativa }^{14} \\
\end{array}$ & 6 & $\begin{array}{c}12 \text { Idosos com diagnóstico clínico de Doença } \\
\text { de Alzheimer/ Instituição de Longa } \\
\text { Permanência de Idosos - Maceió/AL. }\end{array}$ & Brasil & Musicoterapia \\
\hline 05 & $\begin{array}{c}\text { Estudo controlado, } \\
\text { randomizado, simples } \\
\text { cego }^{15}\end{array}$ & 2 & $\begin{array}{l}41 \text { Participantes com DA leve/ Clínica Memória } \\
\text { de Psicogeriatria unidade do Instituto de } \\
\text { Psiquiatria - São Paulo. }\end{array}$ & Brasil & $\begin{array}{c}\text { Reabilitação } \\
\text { Cognitiva } \\
\text { Multidisciplinar } \\
\end{array}$ \\
\hline 06 & $\begin{array}{l}\text { Estudo controlado, } \\
\text { não- cego e sem } \\
\text { randomização }^{16}\end{array}$ & 3 & $\begin{array}{c}32 \text { Pacientes com DA leve a moderada/ Unesp - } \\
\text { Universidade Estadual Paulista }\end{array}$ & Brasil & $\begin{array}{l}\text { Programa de } \\
\text { Intervenção } \\
\text { Motora }\end{array}$ \\
\hline 07 & $\begin{array}{l}\text { Estudo experimental, } \\
\text { controlado, não } \\
\text { randomizado }\end{array}$ & 3 & $\begin{array}{l}59 \text { Pacientes com diagnóstico de demência } \\
\text { (DA, demência vascular ou mista) / Clínica de } \\
\text { Psiquiatria Geriátrica Ambulatorial de uma } \\
\text { escola médica pública brasileira. }\end{array}$ & Brasil & $\begin{array}{c}\text { Programa de } \\
\text { Atividade Física }\end{array}$ \\
\hline 08 & $\begin{array}{l}\text { Pesquisa quantitativa } \\
\text { não aleatória }{ }^{18}\end{array}$ & 4 & $\begin{array}{l}11 \text { Idosos com diagnóstico de demência do tipo } \\
\text { Alzheimer/ Universidade Federal Fluminense } \\
\text { (EPIGG/UFF), Niterói - Rio de Janeiro. }\end{array}$ & Brasil & $\begin{array}{l}\text { Estimulação } \\
\text { Cognitiva }\end{array}$ \\
\hline 09 & $\begin{array}{c}\text { Estudo controlado, } \\
\text { randomizado e duplo } \\
\text { cego }^{19} \\
\end{array}$ & 2 & $\begin{array}{l}39 \text { Participantes com DA grave, nível 3/ Hospital } \\
\text { Demência Especial na cidade de Kobe. }\end{array}$ & Japão & Musicoterapia \\
\hline 10 & $\begin{array}{l}\text { Estudo longitudinal, } \\
\text { controlado, } \\
\text { randomizado e } \\
\text { simples- cego }\end{array}$ & 2 & $\begin{array}{l}98 \text { Pacientes idosos com demência/Lar de } \\
\text { idosos em Mittelfranken - Baviera. }\end{array}$ & Alemanha & $\begin{array}{l}\text { Terapia MAKS } \\
\text { (Estimulação } \\
\text { motora, } \\
\text { atividades de vida } \\
\text { diária, } \\
\text { estimulação } \\
\text { cognitiva e } \\
\text { elemento } \\
\text { espiritual) }\end{array}$ \\
\hline
\end{tabular}

Fonte: Elaborado pelos autores para fins deste estudo.

Após a análise dos artigos selecionados, os métodos não farmacológicos utilizados no tratamento da DA, identificados nos estudos, foram agrupados em três categorias, a saber: 1 . Intervenção motora; 2 . Intervenção cognitiva e 3. Musicoterapia. Ressalta-se que um método pode aparecer em duas categorias distintas por possuir características de ambas as categorias.

\section{Intervenção na atividade motora}

A intervenção motora como um método de tratamento não farmacológico foi utilizada em cinco estudos. $O$ primeiro estudo baseou-se na avaliação da efetividade dos exercícios aeróbicos (esteira) na capacidade cognitiva e funcional de 20 idosos com demência, sendo 16 diagnosticados com DA e quatro com demência mista, recrutados por psiquiatras treinados a 
partir do Centro para a Doença de Alzheimer no Instituto de Psiquiatria da Universidade Federal do Rio de Janeiro (IPUB / UFRJ). Os pacientes foram divididos em grupo exercício (10 pacientes) submetidos à caminhada na esteira, por 30 minutos, duas vezes por semana, durante três meses e grupo controle (10 pacientes) que mantiveram sua terapia farmacológica e clínica, ao longo do tempo do estudo. A avaliação cognitiva foi realizada a partir do Exame

Cambridge Cognitive (CAMCOG), a capacidade funcional foi avaliada pelos testes de Estática e dinâmica saldo Berg Balance Scale (BERG), o "Timed Up and Go" test (TUGT), o teste Sit-toStand (STS), Teste ergométrico e eletrocardiograma de repouso (ECG). Ao final do estudo, observou-se uma melhora estatisticamente significativa no grupo exercício em comparação ao grupo controle no seu estado cognitivo geral e na mobilidade ${ }^{(11)}$.

Já o estudo transversal avaliou a relação entre a prática de atividades físicas e a manutenção da cognição e das atividades de vida diária em 37 pacientes com diagnóstico de DA, leve a moderada, que frequentavam um centro de tratamento dia e outros recrutados em um programa de educação física para a manutenção da independência em AVD.

Os idosos foram divididos em grupo controle (19 pacientes) que realizavam uma hora de exercícios físicos em grupo, duas vezes por semana, por um período de seis meses, DA sedentário (11 pacientes) que não praticavam nenhuma atividade física regular por, pelo menos seis meses e DA ativos ( 7 pacientes) que realizavam sessões de fisioterapia com exercícios respiratórios, treinamento de equilíbrio estático e dinâmico, circuitos de marcha com e sem obstáculos, estimulação de AVD's, da coordenação motora fina e equilíbrio, além de sessões de estimulação cognitiva, com duração de uma hora, duas vezes por semana, por um período de seis meses.

Para avaliação cognitiva e funcional foram utilizados os métodos: Escala Cornell para Depressão em Demência, Clinical Dementia Rating (CDR), o Miniexame do Estado Mental (MMSE), O POMA-Brasil, o "Timed Up and Go Test" (TUGT), as escalas projetadas por Lawton e Brody para AVD's e AIVD's. O estudo demonstrou que os pacientes com DA apresentaram melhorias em relação à mobilidade e AVD em comparação com o grupo sedentário, o que demonstrou que os exercícios físicos apresentam influência positiva no estado cognitivo e funcional de pacientes com $D A^{(12)}$.

Em outra pesquisa, realizada em São Paulo, os autores conduziram um estudo controlado, sem randomização e analisaram a efetividade de um programa de atividades físicas nas funções cognitivas, equilíbrio e risco de quedas em idosos com demência de Alzheimer. $O$ estudo foi realizado com 16 idosos com diagnóstico de DA selecionados por indicação médica e voluntariado, residentes na comunidade de Rio Claro (SP), divididos em grupo intervenção (9 participantes) que realizaram um programa de atividade física regular, sistematizado e supervisionado, três vezes por semana, em dias não consecutivos, com duração de 60 minutos por sessão, durante seis meses, executadas em grupo, com auxílio de estagiários e grupo rotina (7 participantes) que mantiveram suas atividades diárias normalmente. Foram avaliados por instrumentos como a Escala De Equilíbrio Funcional de Berg (EEFB), os testes "Timed up and Go (TUG) avaliados em segundos (TUGs) e passos (TUGp) e o de Agilidade e Equilíbrio Dinâmico (AGILEQ), da bateria de testes da American Alliance for Health Physical Education, Recreation and Dance utilizados para avaliação do equilíbrio, enquanto que para a avaliação cognitiva, utilizouse o Miniexame do Estado Mental (MEEM). Foi observado que a atividade física é um método eficaz para a manutenção das funções cognitivas e equilíbrio, além de prevenção do risco de quedas em idosos com DA, visto que, resultados dos testes demonstraram melhoras significativas no grupo intervenção e piora no grupo rotina ${ }^{(13)}$.

Ainda, nesse sentido, em um estudo experimental, os autores investigaram a atenuação de sintomas neuropsiquiátricos e a redução da carga de cuidadores em pacientes com DA, a partir de um programa de atividades físicas. Participaram do estudo 59 pacientes com diagnóstico de DA, provável demência vascular, ou misturados, e seus cuidadores. Os pacientes dos três grupos de demência foram divididos em níveis mais baixos e mais altos de atividade física e esta foi considerada sistematizada quando realizada pelo menos duas vezes por semana por 30 minutos ou mais e por, pelo menos, seis meses. Utilizaram-se instrumentos como a escala CDR, Secção Cognitiva do Exame Cambridge para Transtornos Mentais do Idoso (CAMCOG), o Inventário Neuropsiquiátrico (NPI), o 
Questionário Mini-sleep (MSQ) e Questionário Baecke Modificado para Idosos (MBQE). O estudo demonstrou que, em geral, os pacientes apresentavam um nível relativamente baixo de atividade física. Aqueles com diagnóstico de DA demonstraram possuir um maior nível de atividade e, consequentemente, menos perturbações neuropsiquiátricas em comparação com aqueles com níveis mais baixos de atividade física, reduzindo assim a carga de seus cuidadores $^{(17)}$.

Por fim, foi realizado um estudo controlado que avaliou o impacto do exercício aeróbico sobre os sintomas neuropsiquiátricos de 32 participantes com diagnóstico provável de DA leve a moderada e a redução da carga e estresse de seus cuidadores, realizada na Universidade Estadual Paulista (UNESP). Foram utilizados instrumentos para avaliação da atividade cognitiva como o Miniexame do Estado Mental (MEEM), o Inventário Neuropsiquiátrico (NPI), além da Escala Cornell de Depressão em Demência. Para o grupo de tratamento, exercícios foram realizados, durante um período de seis meses, 60 minutos por sessão, três vezes por semana em dias não consecutivos e para o grupo controle foram mantidas as atividades normais de vida diária. Ao final do estudo, foi observada uma redução nos sintomas neuropsiquiátricos (agitação / agressividade, depressão, ansiedade, apatia / indiferença, desinibição, irritabilidade e alterações do apetite) entre os participantes do grupo intervenção e um aumento desses sintomas no grupo de controle. Em relação à sobrecarga do cuidador, observou-se uma redução significativa do estresse no grupo intervenção, em comparação ao grupo controle que manteve índices semelhantes ao do inicio do estudo $^{(16)}$.

$\mathrm{Na}$ presente pesquisa, a metade dos estudos abordaram a intervenção motora como um método eficiente de tratamento não farmacológico, por meio de atividades físicas aeróbicas, caminhadas, treinamento de marcha e equilíbrio, dentre outros. Esses estudos, em sua maioria, observaram evoluções significativas nos pacientes submetidos aos programas de atividades físicas, com redução do risco de quedas, melhora no equilíbrio e marcha, melhor desempenho das atividades de vida diária, diminuição de sintomas neuropsiquiátricos e benefícios no desenvolvimento cognitivo e motor.
Estudos têm demonstrado o efeito benéfico de treinamento físico na qualidade de vida, sintomas depressivos, atividades de vida diária e função cognitiva e motora de pacientes com DA. O exercício físico atenua o risco de incapacidade associada aos distúrbios comportamentais em pacientes com demência ${ }^{(21-}$ ${ }^{22)}$. Além de todos esses efeitos positivos, a atividade física também contribuiu de forma significativa para a redução de comportamento agressivo em pacientes com DA quando comparados a um grupo controle ${ }^{(11)}$.

Nos estudos avaliados as principais limitações foram em relação à padronização do tempo de exercício adotado ora com um tempo maior ora com tempo menor. Dentre outras limitações apontadas foram incluídas a falta de controle da intensidade do exercício adotado, a amostra reduzida, estudos transversal sem acompanhamento e ausência de controle de efeitos dos medicamentos psicotrópicos refletidos na cognição.

\section{Intervenção cognitiva}

Nesta revisão de literatura, somente dois estudos que abordavam a intervenção cognitiva como um método de tratamento não farmacológico da DA e um estudo que utilizou uma terapia multicomponente com uma abordagem cognitiva.

Em um estudo controlado, foram avaliados os benefícios de um programa de estimulação multifuncional na cognição, sintomas neuropsiquiátricos e qualidade de vida de 41 pacientes com DA leve e seus cuidadores. Os pacientes e controles foram submetidos a uma avaliação clínica cognitiva e utilizaram-se instrumentos como o Miniexame do Estado Mental (MEEM), Teste de Curto Cognitiva (SKT), Escala de Depressão Geriátrica (GDS), Avaliação da Qualidade de Vida na Escala de Doença de Alzheimer (QoL-AD). A intervenção foi realizada em sessões de grupo com 12 participantes cada, duas vezes por semana, com duração média de 90 minutos por sessão. Foram realizadas atividades de reabilitação cognitiva, treinamento cognitivo, fonoaudiologia, terapia ocupacional, terapia da arte, cultura física, fisioterapia e estimulação cognitiva com leitura e lógica/jogos. Os cuidadores receberam acompanhamento psicológico em sessões grupais, duas vezes por semana, com o objetivo de esclarecer questões pertinentes à DA de seus pacientes/familiares. $O$ 
grupo controle, por sua vez, recebeu atendimento ambulatorial padrão. 0 estudo revelou que o programa de reabilitação cognitiva proporcionou melhoras suaves na cognição dos pacientes do grupo da intervenção, enquanto que os pacientes do grupo controle possuíram uma piora significativa na atenção e global ${ }^{(15)}$.

Ainda, nesse sentido, uma pesquisa descreveu e analisou a eficácia de oficinas de estimulação cognitiva para idosos com demência de Alzheimer. $O$ estudo contou com uma amostra de 12 pacientes com diagnóstico clínico de DA. As sessões ocorreram semanalmente, com duração média de 50 minutos cada, com atividades como técnicas de orientação para a realidade, recursos mnemônicos, reminiscências, atividades de vida diária e atividades de socialização, que estimulavam os déficits apresentados pelos pacientes. Utilizaram-se instrumentos como o Miniexame do Estado Mental (MEEM), Teste do Relógio, Teste de Evocação de Palavras, Escala de Depressão Geriátrica (EDG), Escala de Atividade Instrumental de Vida Diária (AIVD) e Escala de Atividade de Vida Diária Katz (AVD) para a realização do rastreio cognitivo. Ao final do estudo, observou-se que os pacientes participantes nas atividades de estimulação cognitiva apresentaram, em sua maioria, estabilização em seus sintomas clínicos, o que demonstra o efeito benéfico da terapia em pacientes com $D A$, visto que, não houve progressão da doença ${ }^{(18)}$.

Um estudo utilizou a terapia multicomponente conhecida como MAKS que se constitui em tarefas organizadas em três categorias: $M$ - Estimulação motora, A Atividade de Vida Diária, K - Estimulação Cognitiva, precedidos por uma breve introdução chamada de S- Elemento Espiritual. Os autores realizaram um estudo que avaliou a efetividade dessa terapia em pacientes com demência provenientes de cinco lares alemães.

A terapia contou com a participação de 98 pacientes, sendo 48 pacientes alocados no grupo controle e 50 alocados no grupo intervenção, que ocorreu seis vezes por semana e obteve duração de doze meses. As sessões consistiam na introdução com cerca de 10 minutos, seguidos de 30 minutos de exercícios motores como boliche, equilíbrio de uma bola de tênis em um frisbee e trinta minutos de tarefas cognitivas com lápis e papel e foi finalizado com 40 minutos de atividades de vida diária, envolvendo tarefas criativas, com intervalo de 10 minutos entre as atividades. O grupo controle recebeu seus cuidados diários habituais. Foram utilizados os instrumentos como a Escala de Alzheimer Disease Assessment (ADAS-Cog), o Teste de Erlangen da AVD (teste E-AVD) e a subescala humor de NOSGER. Os resultados demonstraram um efeito significativo da Terapia MAKS sobre a função cognitiva em pacientes com demência e sobre a sua capacidade de realizar AVD's em comparação ao grupo controle que recebeu os cuidados habituais, permitindo assim uma maior independência de pacientes com demência leve, durante pelo menos um período de doze meses, sem efeitos adversos ${ }^{(20)}$.

Em relação à cognição, a demência é uma síndrome que impõe barreiras cognitivas que interferem nas atividades de vida diária do indivíduo, diminuindo cada vez mais a sua independência para a realização de cuidados básicos. Sendo assim, a estimulação ou a reabilitação cognitiva desenvolvida, por meio de realização de atividades de memória e de exercício de funções cognitivas são essenciais para que não haja uma progressão da doença ou para que se tenha uma adequação à nova condição de vida que permite que o idoso possa atingir um nível ideal de desempenho físico, psicológico e social, reduzindo assim a sua dependência e, consequentemente, a carga do cuidador, além de melhorar a qualidade de vida de ambos ${ }^{(15,18)}$.

A terapia multicomponente conhecida pela sigla MAKS que submetia os pacientes a atividades de acordo com seu nível de compreensão, que avalia o exame de estado mental demonstrou um efeito positivo sobre a cognição e sobre a capacidade de realizar AVD's, ou seja, as terapias multicomponentes que envolvem estimulação tanto motora quanto cognitiva, podem ser uma opção viável no tratamento não farmacológico da DA, uma vez que são de fácil aplicação, baixo custo e apresentam resultados benéficos aos seus participantes ${ }^{(20,23)}$.

As limitações desses estudos foram o número reduzido em grupo controle, investimento expressivo em recursos humanos para o desenvolvimento do estudo, amostra pequena, assiduidade dos participantes, falta de fomento para estudo e auxílio para os participantes continuar a participação no estudo. 


\section{Musicoterapia}

Em relação ao uso da musicoterapia, dois estudos observaram os efeitos desse método de tratamento não farmacológico como alternativa para pacientes com DA. Em um estudo de abordagem qualitativa descritivo-exploratória, observaram-se os efeitos da música, em cinco pacientes idosos com DA de uma Instituição de Longa Permanência de Idosos, localizada em Maceió/AL. Foi utilizado um formulário de entrevista semiestruturado e um diário de campo.

As entrevistas foram realizadas antes e após as cinco sessões totais de música que possuíam duração de 20 a 45 minutos, aproximadamente, de acordo com as preferências pessoais do idoso, dessa forma, cada participante possuía um $C D$ personalizado composto por cinco músicas. Os resultados demonstraram o efeito benéfico da música em pacientes com diagnóstico de $D A$, visto que houve um resgate de memórias e experiências vividas por esses pacientes, também foi observada a expressão de sentimentos como felicidade e saudade percebidos na fisionomia apresentada, além desses pacientes relatarem uma redução da dor quando sob influência das músicas ${ }^{(14)}$.

Em um estudo controlado, randomizado e duplo cego foram avaliadas as diferenças entre os efeitos em curto e longo prazo de abordagens passivas e interativas, utilizando música individualizada associada a memórias pessoais em idosos com demência grave. Participaram do estudo 39 idosos com diagnóstico de DA grave, avaliados por meio do Miniexame do Estado Mental (MEEM), divididos em três grupos, sendo um grupo controle que passava um tempo com seu cuidador e sem a intervenção de música, um grupo música passiva que ouviu a música selecionada por meio de um leitor de $C D$, um grupo música interativa que além de ouvir a música por um leitor de $C D$, participava de sessões interativas (cantando, dançando, batendo palmas) com o auxilio de um facilitador.

A intervenção possuiu uma duração de 30 minutos cada, uma vez por semana, durante 10 semanas, constituindo 10 sessões totais. Para avaliação dos efeitos de curto prazo foi utilizada a Escala de Faces e em longo prazo a Escala de Avaliação de Alzheimer (BEHAVE-AD). Ao final do estudo, foi observada uma redução do estresse, um relaxamento, além de evocação de sentimentos de felicidade e contentamento nos pacientes participantes da intervenção música passiva e interativa, tanto em curto quanto em longo prazo, em comparação ao grupo controle sem música. O grupo controle, por sua vez, apresentou uma deterioração dos sintomas psicológicos e comportamentais ao final do período de intervenção ${ }^{(19)}$.

A musicoterapia é uma forma de tratamento não farmacológico para a $\mathrm{DA}$, que pode permitir a trazer a recordação de momentos felizes proporcionar agradáveis estados emocionais, redução do estresse e aumento do relaxamento.

Nesse sentido, a terapia com música é uma forma do idoso entrar em contato com seu passado por meio da evocação de memórias, potencialidades, fortalecimento da autoestima e identidade. Ainda, a musicoterapia pode ser usada por profissionais de saúde, no cuidado ao paciente portador do Alzheimer de forma a proporcionar o bem-estar desses indivíduos, amenizando seu sofrimento psicossocial. Além de todos esses benefícios, a terapia com música atua de modo a promover a reinserção desses indivíduos à sociedade, proporcionando uma maior comunicação e interação social desses pacientes dentro do seu ambiente de convívio, sendo assim um mecanismo eficaz no retardo da progressão da demência ${ }^{(8,24)}$.

As limitações dos estudos analisados foram o tempo de intervenção, duração, comparação apenas com a música, amostra reduzida e indivíduos com demência grave.

Diante do exposto, observa-se que os profissionais de saúde exercem função primordial no cuidado ao paciente com DA. A exemplo, o enfermeiro age de forma a promover um suporte aos cuidadores e familiares quanto às transformações cognitivas e funcionais ocasionadas por essa enfermidade, apresentando a melhor forma de enfrentamento destas ${ }^{(21-29)}$. Esses profissionais também têm a função de propiciar o cuidado sempre estimulando a manutenção da autoestima e autonomia desses pacientes, utilizando-se de ferramentas, como os métodos não farmacológicos, para o tratamento, visando assim, a minimizar os efeitos indesejáveis atrelados à doença e possibilitar uma melhor qualidade de vida ${ }^{(8,9,24)}$.

Após a explanação dos métodos existentes para o tratamento não farmacológico da DA e sua eficácia na atenuação de sintomas cognitivos e 
motores de pacientes com demência ${ }^{(25,27,30-31)}$. Observa-se que não existe atualmente um tratamento que possa reverter totalmente a deterioração do funcionamento cognitivo e motor causados pela DA, eles apenas visam a retardar a progressão da doença ${ }^{(25-27)}$.

Por fim, observa-se que os resultados apontados nesta revisão podem contribuir para a prática de enfermagem. Diante dos métodos não farmacológicos discutidos, atenta-se para a adoção de intervenções de enfermagem que beneficiem a qualidade de vida do idoso, tais como atividades motoras, terapias alternativas multiprofissionais como musicoterapia, com vistas a promover as atividades de vida diária desses pacientes que convivem com a DA.

\section{CONSIDERAÇÕES FINAIS}

A revisão integrativa da literatura permitiu o desenvolvimento de um estudo mais aprofundado acerca dos métodos não farmacológicos para o tratamento da DA. As intervenções mais abordadas, ou seja, com um maior número de produções científicas foram as que envolviam atividades motoras, em razão da sua facilidade de implementação, baixo custo e por apresentarem resultados benéficos aos pacientes com DA.

Além destas, também foram encontrados estudos que utilizavam as intervenções cognitivas e terapia com música como forma de tratamento e que provaram ser eficientes, pois reduziram a dependência para a realização de atividades de vida diária e, consequentemente, melhoraram a qualidade de vida desses pacientes.

As principais limitações desta revisão foram a análise de estudos que não tiveram controle de algumas variáveis como intensidade e tempo da atividade física, amostra reduzida, não comparação entre música e outras intervenções e estudos transversais. Assim exposto, sugere-se que outra revisão seja realizada com estudos que tiveram tempo similar de atividade física, com amostras maiores e comparação da musicoterapia com outro tipo de intervenção.

Em resumo, foi possível observar que existem muitas lacunas no conhecimento acerca dessa temática, uma vez que a utilização desses métodos é, ainda, pouco usual. Portanto, faz-se necessário o desenvolvimento de um maior número de pesquisas acerca das intervenções não farmacológicas, sua forma de implementação, seus riscos e benefícios.

\section{REFERÊNCIAS}

1. Bernardo LD. Idosos com doença de Alzheimer: Uma revisão sistemática sobre a intervenção da terapia ocupacional nas alterações em habilidades de desempenho. Cad Bras Ter Ocup. 2018;26(4):926-42. DOI: 10.4322/2526-8910.ctoar1066

2 World Health Organization (WHO). World Alzheimer Report 2018. Genebra: WHO; 2018.

3. Boff MS, Sekyia FS, Bottino CMC. Revisão sistemática sobre prevalência de demência entre a população brasilieira. Rev Med. 2015;94(3):15461. DOI: 10.11606/issn.1679-9836.v94i3p154-161 4. Teixeira JB, Souza Junior PBR, Higa J, Theme Filha MM. Doença de Alzheimer: Estudo da mortalidade no Brasil, 2000-2009, 2000-2009. Cad Saúde Pública 2015;31(4):850-60. DOI: 10.1590/0102-311X00144713

5. Cazarim MS, Moriguti JC, Ogunjimi AT, Pereira LRL. Perspectives for treating Alzheimer's disease: A review on promising pharmacological substances. São Paulo Med J. 2016;134(4):342-54. DOI: $10.1590 / 1516-3180.2015 .01980112$

6. Alberca JMG. Cognitive intervention therapy as treatment for behaviour disorders in Alzheimer disease: Evidence on efficacy and neurobiological correlations. 2015;30(1):8-15. DOI: $10.1016 /$ j.nrl.2012.10.002

7. Engelhardt E. Tratamento da doença de Alzheimer: Recomendações e sugestões do Departamento Científico de Neurologia Cognitiva e do Envelhecimento da Academia Brasileira de Neurologia. Arq Neuro-Psiquiatr. 2015;63(4):1104-1112. DOI: 10.1590/S0004282X2005000600035

8. Sánchez-Valdeón L, Fernández-Martínez E, Loma-Ramos S, López-Alonso Al, Bayón Darkistade E, Ladera V. Canine-assisted therapy and quality of life in people with alzheimer's-type dementia: Pilot study. Front Psychol. 2019;10:1332. DOI: 10.3389/fpsyg.2019.01332

9. Sun X, Zhou X, Zhang Y, Liu H. Reporting and methodological quality of systematic reviews and meta?analyses of nursing interventions in patients with alzheimer's disease: General implications of the findings. J Nurs Scholarsh. 2019;51(3):308-16. DOI: 10.1111/jnu.1246

10. Pedrosa KKA, Oliveira ICM, Feijão AR, Machado RC. Enfermagem baseada em evidencia: Caracterização dos estudos no Brasil. Cogitare Enferm. 2015;20(4):733-41. DOI: $\underline{10.5380 / c e . v 20 i 4.40768}$ 
11. Arcoverde $\mathrm{C}$, Moraes $\mathrm{H}$, Almeida $\mathrm{C}$, Araujo NB, Vasques PE, Silveira $H$, et al. Treadmill training as an augmentation treatment for Alzheimer's disease: A pilot randomized controlled study. Arq Neuropsiquiatr. 2014;72(3):190-6. DOI: 10.1590/0004282X20130231

12. Arcoverde C, Deslandes A, Rangel A, Rangel A, Pavão $R$, Nigri $F$, et al. Papel da atividade física na manutenção da cognição e atividades de vida diária em idosos com doença de Alzheimer. Arq Neuro-Psiquiatr. 2008;66(2b):323-7. DOI: 10.1590/S0004282X2008000300007

13. Hernandez SSS, Coelho FGM, Gobbi S, Stella F. Efeitos de um programa de atividade física nas funções cognitivas, equilíbrio e risco de quedas em idosos com demência de Alzheimer. Rev Bras Fisioter. 2010;14(1):68-74. DOI: 10.1590/S1413-35552010000100011

14. Albuquerque MCS, Nascimento LO, Lyra ST, Figueredo MCS, Brêda MZ. Os efeitos da música em idosos com doença de Alzheimer de uma instituição de longa permanência. Rev Eletr Enferm. 2012;14(2):404-13.

DOI: 10.5216/ree.v14i2.12532

15. Viola LF, Nunes PV, Yassuda MS, Aprahamian I, Santos FS, Santos GD, et al. Effects of a multidisciplinar cognitive rehabilitation program for patients with mild Alzheimer's disease. Clinics 2011;66(8):1395-1400. DOI: 10.1590/S1807-59322011000800015

16. Stella F, Canonici AP, Gobbi S, Galduroz RF, Cação JC, Gobbi LT. Attenuation of neuropsychiatric symptoms and caregiver burden in Alzheimer's disease by motor intervention: $A$ controlled trial. Clinics 2011;66(8):1353-60. DOI: 10.1590/S1807-59322011000800008

17. Christofoletti G, Oliani MM, BuckenGobbi LT, Gobbi S, Beinotti F, Stella F. Physical activity attenuates neuropsychiatric disturbances and caregiver burden in patients with dementia. Clinics 2011;66(4):613-8. DOI: 10.1590/S180759322011000400015

18. Souza PA, Bastos RCS, Santana RF, Sá SPC, Cassiano KM. Oficinas de estimulação cognitiva para idosos com demência: Uma estratégia de cuidado na enfermagem gerontológica. Rev Gaúcha Enferm. 2008;29(4):588-95. DOI: 10.5335/rbceh.2012.305

19. Sakamoto $M$, Ando $H$, Tsutou $A$. Comparing the effects of different individualized music interventions for elderly individuals with severe dementia. Int Psychogeriatr. DOI: 2013;25(5):775-84. 10.1017/S1041610212002256 20. Graessel E, Stemmer R, Eichenseer B, Pickel S, Donath C, Kornhuber J pharmacological, multicomponent group therapy in patients with degenerative dementia: A 12-month randomized, controlled trial. BMC Med. 2011;9:129. DOI: 10.1186/1741-7015-9-129

21. Toots A, Wiklund R, Littbrand $H$, Nordin $E$, Nordström $\mathrm{P}$, Lundin $\mathrm{OL}$, et al. The effects of exercise on falls in older people with dementia living in nursing homes: A randomized controlled trial. J Am Med Dir Assoc. 2019;20(7):835-42. DOI: 10.1016/j.jamda.2018.10.009

22. Henskens M, Nauta IM, Van Eekeren MCA, Scherder EJA. Effects of physical activity in nursing home residents with dementia: $A$ randomized controlled trial. Dement Geriatr Cogn Disord. 2018;46(1/2):60-80. DOI: $\frac{10.1159 / 000491818}{Y u F, \text { Chen } Y,}$

23. Yu F, Chen Y, Mathiason MA, Wan Q, Lin FV. Cognitive and physical factors affecting daily function in Alzheimer's disease: A cross-sectional analysis. Nurs Health Sci. 2019;21(1):14-20. DOI: $10.1111 /$ nhs. 12426

24. Matyas N, Keser AF, Wagner G, Teufer B, Auer $\mathrm{S}$, Gisinger $\mathrm{C}$, et al. Continuing education for the prevention of mild cognitive impairment and Alzheimer's-type dementia: A systematic review and overview of systematic reviews. BMJ Open. 2019;9:1-10. DOI: $10.1136 /$ bmjopen-2018$\underline{027719}$

25. Muelle AR, Rodriguez MML. Dance for people with alzheimer's disease: A mini-review. Curr Alzheimer Res. 2019;25(1):1-6. DOI: 10.2174/1567205016666190725151614

26. Evangelista LB, Souza MMT. Nursing on overload experienced by patients with Alzheimer's caregiver. Rev Pró-UniverSUS. 2015 [citado em 15 jan 2018]; 6(1):17-21. Disponível em:

http://editorauss.uss.br/index.php/RPU/article/vi ew/402/473

27. Areias BB, Bonfim MM, Schiaveto FB. A participação da enfermagem frente ao cuidador de idosos portadores de Alzheimer. Revista Fafibe. 2015 [citado em 15 jan 2018]; 8(1):44-63. Disponível em: http://unifafibe.com.br/revistasonline/arquivos/rev istafafibeonline/sumario/36/30102015183428.pdf

28. Wong R, Amano T, Lin SY, Zhou Y, Morrow-Howell N. Strategies for the recruitment and retention of racial/ethnic minorities in 
alzheimer disease and dementia clinical research. Curr Alzheimer Res. 2019;16(5):458-71. DOI: 10.2174/1567205016666190321161901

29. Glinz D, Gloy VL, Monsch AU, Kressig RW, Patel C, McCord KA, et al. Acetylcholinesterase inhibitors combined with memantine for moderate to severe Alzheimer's disease: A metaanalysis. Swiss Med Wkly. 2019;17(3):149-55. DOI: $\frac{10.4414 / \text { smw.2019.20093 }}{\text { Swn } X \text {. }}$

30. Sun $X$, Zhou X, Yu Y, Liu H. Exploring reporting quality of systematic reviews and Metaanalyses on nursing interventions in patients with Alzheimer's disease before and after PRISMA introduction. BMC Med Res Methodol. 2018;18(2):158-61. DOI: 10.1186/s12874-018$\underline{0622-7}$

31. King JB, Jones KG, Goldberg E, Rollins $M$, MacNamee $K$, Moffit $C$, et al. Increased functional connectivity after listening to favored music in adults with alzheimer dementia. J Prev Alzheimers Dis. 2019;6(1):56-62. DOI: 10.14283/ipad.2018.19

Nota: Trabalho de monografia de final de curso de graduação em enfermagem da Universidade de Brasília UNB/FCE. Este artigo não foi financiado por uma agência de financiamento.

Recebido em: 22/02/2018

Aprovado em: 24/10/2019

Endereço de correspondência:

Marina Morato Stival

Endereço: Curso de Enfermagem da Universidade de Brásilia/Faculdade de Ceilândia, Campus Universitário Centro Metropolitano, Ceilândia Sul.

CEP: 72220-275 - Brasília/DF - Brasil

E- mail: marinamorato@unb.br 